\title{
Demonstration of Functional Similarity of Proposed Biosimilar ABP 501 to Adalimumab
}

\author{
Jyoti Velayudhan $^{1} \cdot$ Yuh-feng Chen ${ }^{1}$ Amanda Rohrbach ${ }^{1}$ - Christina Pastula ${ }^{1}$. \\ Gwen Maher $^{1} \cdot$ Heather Thomas ${ }^{1} \cdot$ Ryan Brown $^{1} \cdot$ Teresa L. Born ${ }^{1}$
}

Published online: 15 July 2016

(c) The Author(s) 2016. This article is published with open access at Springerlink.com

\begin{abstract}
Background Due to the complex molecular structure and proprietary manufacturing processes of monoclonal antibodies (mAbs), differences in structure and function may be expected during development of biosimilar mAbs. Important regulatory requirements for approval of biosimilar products involve comprehensive assessments of any potential differences between proposed biosimilars and reference mAbs, including differences in all known mechanisms of action, using sensitive and relevant methods. Any identified structural differences should not result in differences in biofunctional or clinical activity.

Objective A comprehensive assessment comparing the Amgen biosimilar candidate ABP 501 with FDA-licensed adalimumab (adalimumab [US]) and EU-authorized adalimumab (adalimumab [EU]) was conducted to demonstrate similarity in biofunctional activity.

Methods The functional similarity assessment included testing of binding kinetics to soluble tumor necrosis factor $\alpha(\mathrm{TNF} \alpha)$ and relative binding to transmembrane TNF $\alpha$. The neutralization of TNF $\alpha$-induced caspase activation, $\mathrm{TNF} \alpha-$ and lymphotoxin- $\alpha$ (LT $\alpha$ )-induced chemokine production, and cytotoxicity was also tested. Binding to Fcgamma receptors Fc $\gamma$ RIa, Fc $\gamma$ RIIa $(131 \mathrm{H})$, Fc $\gamma$ RIIIa (158V and $158 \mathrm{~F})$, and neonatal $\mathrm{Fc}$ receptor $(\mathrm{FcRn})$ was compared with the reference mAbs, as was antibody-dependent cellmediated cytotoxicity and complement-dependent cytotoxicity.
\end{abstract}

Teresa L. Born

bornt@amgen.com

1 Amgen, Inc., One Amgen Center Drive, Thousand Oaks, CA 91320, USA
Results The data demonstrate that ABP 501 is similar to both adalimumab (US) and adalimumab (EU) with respect to evaluated biofunctional activities.

Conclusion Similarity in biofunctional activity is a critical component of the totality of evidence required for demonstration of biosimilarity. The functional similarity demonstrated for ABP 501 comprehensively assesses the known mechanisms of action of adalimumab, supporting the conclusion that ABP 501, adalimumab (US), and adalimumab (EU) are likely to be clinically similar.

\section{Key Points}

ABP 501, a biosimilar candidate to adalimumab, binds to and neutralizes tumor necrosis factor $\alpha$ $(\mathrm{TNF} \alpha)$, a pleiotropic proinflammatory cytokine that can induce a variety of cellular effects contributing to autoimmune disease.

A comprehensive similarity assessment comparing ABP 501, adalimumab (US), and adalimumab (EU) demonstrated similarity with respect to a variety of biological properties, including binding to soluble and transmembrane TNF $\alpha$; neutralization of TNF $\alpha$-induced caspase activation, TNF $\alpha$ - and lymphotoxin- $\alpha$ (LT $\alpha)$-induced chemokine production, and cytotoxicity; Fc receptor binding; and effector function activation.

Similarity in preclinical biological activity contributes to the foundation of the stepwise approach used to demonstrate biosimilarity, which subsequently includes human pharmacokinetic studies and clinical efficacy and safety studies. 


\section{Introduction}

Biosimilars, biologic products similar in terms of quality, efficacy, and safety to licensed biologic reference products, are being developed to provide less expensive therapeutic alternatives in an effort to reduce healthcare expenditures [1]. The high complexity in molecular structure and unique/proprietary biomanufacturing processes of biologics, however, can result in structural and functional differences, making it impossible to produce biosimilar molecules that are identical to the innovator biologics [2]. It is therefore important to demonstrate that structural and functional differences between biosimilars and reference biologics do not result in clinically meaningful differences in safety or efficacy. The US Food and Drug Administration (FDA) and European Medicines Agency (EMA) have developed guidelines for the development of biosimilars that recommend a stepwise approach emphasizing the totality of evidence for demonstration of biosimilarity, encompassing similarity in analytical studies (physiochemical product quality attributes and biological activity); relevant animal studies (preclinical pharmacokinetics, pharmacodynamics, and toxicity); and finally, clinical studies (pharmacokinetics and pharmacodynamics, immunogenicity, safety, and efficacy) (Fig. 1) [3-5]. Sensitive analytical methods capable of detecting potential differences are the foundation of the stepwise biosimilar evaluation process, and identified differences are further evaluated to confirm they do not impact clinical efficacy and safety.

ABP 501 is being developed as a biosimilar to adalimumab, a human immunoglobulin G1 (IgG1) anti-tumor necrosis factor $\alpha$ (anti-TNF $\alpha$ ) monoclonal antibody (mAb) that prevents interaction of TNF $\alpha$ with its receptors, thereby interfering with the inflammatory signaling central

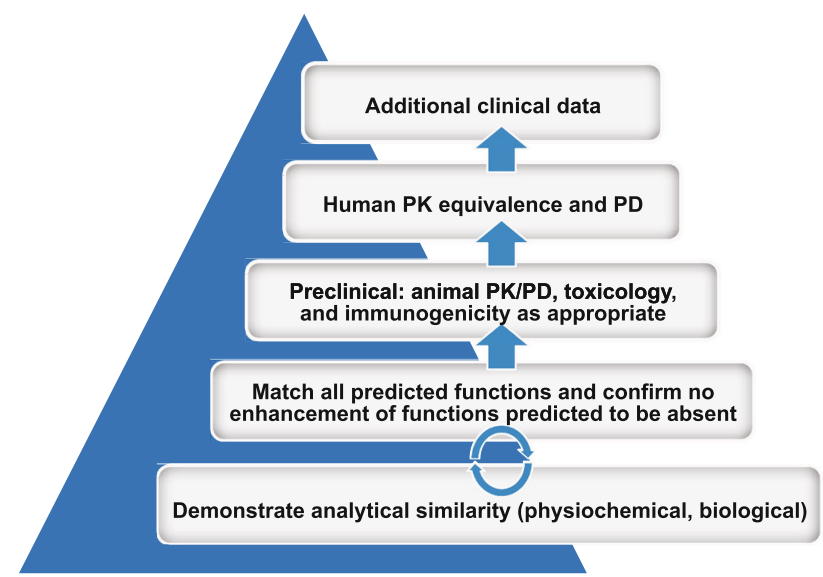

Fig. 1 Stepwise, totality-of-evidence approach to demonstrating biosimilarity [3, 4]. $P D$ pharmacodynamics, $P K$ pharmacokinetics to chronic autoimmune diseases such as rheumatoid arthritis, psoriasis, ulcerative colitis, and Crohn's disease. Adalimumab is highly specific for TNF $\alpha$ and does not bind murine TNF $\alpha$ or the closely related human cytokine lymphotoxin- $\alpha(\mathrm{LT} \alpha)[6,7]$. Adalimumab is known to bind soluble TNF $\alpha(\operatorname{sTNF} \alpha)$ with high affinity and inhibit its bioactivity [7]. The primary bioactivity of TNF $\alpha$ is elicited when the soluble version of the cytokine engages TNF receptor 1 (p55TNFR). More specifically, sTNF $\alpha$ can ultimately induce either nuclear factor kappa B (NFkB)dependent gene expression or (in the absence of NFKB activity) cell death, depending upon the molecular context of the responding cell [8]. The NFKB-dependent induction of gene expression, in particular, is central to the propagation of autoimmune disease pathology.

Adalimumab also binds to uncleaved transmembrane $\mathrm{TNF} \alpha(\mathrm{mbTNF} \alpha)$ and, since it is an IgG1 capable of binding to $\mathrm{Fc}$ gamma receptors $(\mathrm{Fc} \gamma \mathrm{R})$ [7], mediates induction of antibody-dependent cell-mediated cytotoxicity (ADCC) and complement-dependent cytotoxicity (CDC) in vitro [9]. The relevance of ADCC- or CDC-mediated cytotoxicity of mbTNF $\alpha$-expressing cells in relation to clinical efficacy is not well established, but may be important, particularly in inflammatory bowel diseases $[7,10]$. It is well known that differences in the glycosylation pattern within the $\mathrm{Fc}$ region of an IgG monoclonal antibody can have significant effects on the effector functions of the molecule [11]. Since analytical differences, especially in the glycan profiles, may be expected between a biosimilar $\mathrm{mAb}$, other biosimilars, and the reference $\mathrm{mAb}$ product, it is important to fully characterize effector functions of the candidate biosimilar $\mathrm{mAb}$, even if the precise contribution to clinical efficacy is unclear.

In addition to mediating effector functions via $\mathrm{Fc} \gamma \mathrm{R}$ binding, adalimumab is capable of binding to the neonatal Fc receptor $(\mathrm{FcRn})$, which can influence the plasma halflife of the antibody. The amino acid sequences of the $\mathrm{Fc}$ region of IgG1 mAbs are identical; however, there is some evidence that some post-translational modifications, or overall $\mathrm{mAb}$ conformation, may confer differences in FcRn binding affinities and therefore plasma half-lives [7]. Therefore, similarity in FcRn binding is critical for providing confidence that the clinical pharmacokinetic profile will be similar between the two products.

Although ABP 501 and adalimumab share the same amino acid sequence [12], differences could be expected in product quality attributes due to inherent differences in expression systems, bioprocess, and purification [13]. Demonstrating equivalence of all functional properties is of foundational importance during the stepwise development of a biosimilar (Fig. 1). The analytical and functional equivalence will ultimately support abbreviated clinical studies and contribute to the scientific justification for 
extrapolation to all approved indications [13-15]. Results of the analytical similarity assessment comparing ABP 501 to FDA-licensed adalimumab (adalimumab [US]), and EUauthorized adalimumab (adalimumab [EU]) have been previously reported (Liu et al. [16]). The objectives of these studies are to provide a comprehensive assessment of functional similarity, to address the pleiotropic effects induced by TNF $\alpha$ as well as the multitude of interactions mediated by the $\mathrm{Fc}$ region of an IgG1 mAb, and to assess both binding activities and functional outcomes.

\section{Methods}

\subsection{Materials}

The ABP 501 drug product was manufactured by Amgen Inc. (Thousand Oaks, CA, USA). Multiple lots of adalimumab (US) (Humira ${ }^{\circledR}$, AbbVie, North Chicago, IL, USA) and adalimumab (EU) were procured and stored according to the manufacturer's instructions. For ABP 501, drug product was used for all analyses. An ABP 501 reference standard lot was included in most analyses. Recombinant sTNF $\alpha$ was purchased from PeproTech (Rocky Hill, NJ, USA) or R\&D Systems (Minneapolis, MN, USA). Chinese hamster ovary cells expressing non-cleavable mbTNFa have been previously described, and are referred to as MT-3 [17].

\subsection{Tumor Necrosis Factor $\alpha(\mathrm{TNF} \alpha)$ Binding Assays}

Relative binding to $\mathrm{STNF} \alpha$ was determined by solid phase enzyme-linked immunosorbent assay (ELISA). Recombinant STNF $\alpha$ was coated onto the wells of a microtiter ELISA plate and the plate was blocked using a gelatin buffer. Dilutions of reference standard and test samples were added to the appropriate wells and incubated for 90 min at ambient temperature. Bound $\mathrm{mAb}$ was detected with goat anti-human $\operatorname{IgG}$ ( $\mathrm{Fc}$ fragment) conjugated to horseradish peroxidase (HRP). Relative binding activities were calculated based on the ratio of half-maximal effective concentration $\left(\mathrm{EC}_{50}\right.$; concentration at which $50 \%$ inhibition is observed) values of the reference standard curve relative to the test sample. A total of ten lots of each test $\mathrm{mAb}$ were assessed.

The kinetics of binding to recombinant sTNF $\alpha$ were determined by surface plasmon resonance (SPR) using a Biacore $^{\mathrm{TM}}$ T200 (GE Healthcare Bio-Sciences, Piscataway, NJ, USA) with single-cycle kinetics methodology. Goat anti-human IgG Fc-specific capture antibody (Jackson ImmunoResearch, West Grove, PA, USA) was immobilized to the sensor chip, allowing for capture of ABP 501, adalimumab (US), and adalimumab (EU). Soluble TNF $\propto$ was tested at escalating concentrations $(0.18-60 \mathrm{nM})$. The double-referenced data from the single-cycle kinetic run was fitted locally to a 1:1 binding model with Biacore kinetics software. The association rate constant $\left(k_{\mathrm{a}}\right)$, the dissociation rate constant $\left(k_{\mathrm{d}}\right)$, and the dissociation equilibrium binding constant $\left(K_{\mathrm{d}}\right)$ were fitted globally. The relative binding affinity of each of the samples was calculated based on the $K_{\mathrm{d}}$ value as compared with the ABP 501 reference standard. A total of three lots of each test $\mathrm{mAb}$ were assessed.

Binding to mbTNF $\alpha$ was assessed in a competitive cellbased binding assay using MT-3 cells and Alexa Fluor ${ }^{\circledR}$ 488 (Thermo Fisher Scientific, Waltham, MA, USA) dye (Alexa-488)-labeled ABP 501. A dose titration of reference standard, ABP 501, adalimumab (US), or adalimumab (EU) test samples and a fixed concentration of the labeled $\mathrm{mAb}$ were incubated with cells for $4-6 \mathrm{~h}$ at room temperature. Binding was assessed by measuring cellbound fluorescence on an Acumen ${ }^{\circledR}$ eX3 imaging cytometer (TTP Labtech, Hertfordshire, UK), wherein binding of the test mAbs to mbTNF $\alpha$ was reflected in the decreased binding of the labeled mAb. After assessing parallelism of the dose-response curves, the test-sample binding relative to the reference standard was determined using a 4-parameter logistic model fit (SoftMax ${ }^{\circledR}$ Pro Software, Molecular Devices, Sunnyvale, CA, USA). A total of three lots each of ABP 501, adalimumab (US), and adalimumab (EU) were tested, with three independent determinations for each lot.

\subsection{Neutralization of TNF $\alpha$ Bioactivity}

Monocytic (U937) cells were stimulated with $3 \mathrm{ng} / \mathrm{mL}$ $\mathrm{TNF} \alpha$ in the presence of a dose titration of reference standard, ABP 501, adalimumab (US), or adalimumab (EU) for approximately $2 \mathrm{~h}$. Caspase-Glo $3 / 7^{\circledR}$ reagent (Promega, Madison, WI, USA) was added to the samples, with an additional 30- to 60-min incubation. Luminescence, measured in relative luminescence units (RLUs), was determined as a measure of the degree of caspase activation. After assessing parallelism of the dose-response curves, the test-sample activity relative to the reference standard was determined using a 4-parameter logistic model fit (SoftMax Pro). Multiple lots $(\geq 10)$ of ABP 501, adalimumab (US), and adalimumab (EU) were tested, with three independent determinations each.

Human umbilical vein endothelial cells (HUVEC) were cultured in a 96-well culture plate prior to stimulation with $3 \mathrm{ng} / \mathrm{mL} \mathrm{TNF} \alpha$ or $10 \mathrm{ng} / \mathrm{mL} \mathrm{LT} \alpha$ for $4 \mathrm{~h}$ in the presence of a dose titration of ABP 501, adalimumab (US), or adalimumab (EU). Supernatants were collected from each well, and the concentration of interleukin-8 (IL-8) was quantified 
using a single-spot immunoassay (Meso Scale Diagnostics, Rockville, MD, USA). Percent of control (POC) was calculated based on IL- 8 produced in unstimulated cells and in cells that were stimulated in the absence of added test antibody. The $\mathrm{EC}_{50}$ was calculated for each sample using Prism $^{\circledR}$ sigmoidal dose-response-curve-fitting software (GraphPad Software, La Jolla, CA, USA). A total of three lots of each test mAb were compared.

Fibrosarcoma (L929) cells were cultured in 96-well culture plates and sensitized with actinomycin D $(2 \mu \mathrm{g} / \mathrm{mL})$ for $2 \mathrm{~h}$, then stimulated with TNF $\alpha(0.75 \mathrm{ng} / \mathrm{mL})$ in the presence of a dose titration of ABP 501, adalimumab (US), or adalimumab (EU). Following overnight incubation, cytotoxicity was measured using the viability indicator dye alamarBlue $^{\circledR}$ (Invitrogen/Thermo Fisher Scientific, Carlsbad, CA, USA), which was added to the cells during the final $4 \mathrm{~h}$ of stimulation. Plates were analyzed for fluorescence on an EnVision ${ }^{\circledR} 2101$ Multilabel Reader (PerkinElmer, Waltham, MA, USA). Percent viability was calculated based on viability of unstimulated cells and of cells stimulated in the absence of added test antibody. The $\mathrm{EC}_{50}$ was calculated for each sample using sigmoidal doseresponse-curve-fitting software (GraphPad Prism). A total of three lots of each test mAb were compared.

\subsection{Fc Receptor Binding}

Relative binding to human Fc $\gamma$ RIa, Fc $\gamma$ RIIa (131H), and Fc $\gamma$ RIIIa (158V and 158F) was determined by AlphaLISA $^{\circledR}$ (reagents from PerkinElmer, Waltham, MA, USA). Fc $\gamma$ RIa-HIS-GST protein ( $1 \mathrm{nM}$ final), Fc $\gamma$ RIIa $(131 \mathrm{H})-\mathrm{GST}-\mathrm{H} 6$ protein $(2 \mathrm{nM}$ final), Fc $\gamma$ RIIIa (158V)GST-His6 protein (1 nM final), or Fc $\gamma$ RIIIa (158F)-GSTHis6 protein (4 $\mathrm{nM}$ final) were pre-incubated with Glutathione AlphaLISA Acceptor beads biotinylated $\mathrm{IgG1}$ $\mathrm{mAb}$ competitor ( $1 \mathrm{nM}$ final for Fc $\gamma$ RIa, $0.4 \mathrm{nM}$ final for Fc $\gamma$ RIIa, and $2 \mathrm{nM}$ final for Fc $\gamma$ RIIIa), and a dose titration of reference standard, ABP 501, adalimumab (US), and adalimumab (EU). In one experiment, recombinant sTNF $\alpha$ was included at an equimolar concentration. Samples were incubated at ambient temperature for approximately $22 \mathrm{~h}$. Streptavidin-coated donor beads were added to each well and incubated for approximately $22 \mathrm{~h}$. Samples were read for luminescence (in RLUs) with an EnVision plate reader, using an AlphaScreen ${ }^{\circledR}$ protocol (PerkinElmer, Waltham, MA, USA). After assessing parallelism of dose-response curves, the test-sample binding relative to the reference standard was determined using a 4-parameter logistic model fit (SoftMax Pro). Multiple lots $(\geq 10)$ of ABP 501, adalimumab (US), and adalimumab (EU) were tested, with three independent determinations each. A single lot of each test $\mathrm{mAb}$ was assessed for Fc $\gamma \mathrm{RIIIa}(158 \mathrm{~V})$ binding in the presence of sTNF $\alpha$.
Relative binding to FcRn was determined using a competitive image cytometry-based assay using engineered 293T cells (293T-7A1) overexpressing human FcRn. Dose titrations of reference standard, ABP 501, adalimumab (US), and adalimumab (EU) were incubated with 293T7A1 cells and a fixed concentration of recombinant IgG1 Fc labeled with Alexa-488 $(0.1 \mu \mathrm{g} / \mathrm{mL})$ for approximately $4 \mathrm{~h}$ at ambient temperature at $\mathrm{pH} 6$. After incubation, the cell-bound fluorescence was read on an Acumen eX3 imaging cytometer wherein binding of the test mAbs to FcRn was reflected in the decreased binding of the labeled $\mathrm{mAb}$. After assessing parallelism of dose-response curves, the test-sample binding relative to the reference standard was determined using a 4-parameter logistic model fit. Multiple lots $(\geq 10)$ of ABP 501, adalimumab (US), and adalimumab (EU) were tested, with three independent determinations each.

\subsection{Induction of Effector Function}

To determine ADCC activity, MT-3 target cells were labeled with calcein-AM dye (Sigma-Aldrich, St. Louis, MO, USA) prior to incubation with a dose titration of reference standard, ABP 501, adalimumab (US), or adalimumab (EU). Effector cells (NK-92M1 cells stably transfected with human Fc $\gamma$ RIIIa [158V] licensed from Conkwest [Cardiff-by-the-Sea, CA, USA], now NantKwest) were then added to the opsonized target cells at an effector-to-target ratio of 25:1 and incubated for approximately $1 \mathrm{~h}$. Calcein released from lysed target cells was determined by measuring the fluorescence of the supernatant. After assessing parallelism of dose-response curves, the percent cytotoxicity of ABP 501, adalimumab (US), or adalimumab (EU) test samples relative to the reference standard was determined using a 4-parameter logistic model fit (SoftMax Pro). Multiple lots $(\geq 10)$ of ABP 501, adalimumab (US), and adalimumab (EU) were tested, with three independent determinations for each lot.

CDC activity was measured using MT-3 cells. Target cells were labeled with calcein-AM prior to incubation with a dose titration of ABP 501, adalimumab (US), or adalimumab (EU). Baby rabbit complement (Cedarlane, Burlington, ON, Canada) was added to opsonized target cells for approximately $1 \mathrm{~h}$ to allow complement-mediated lysis. Calcein released from lysed target cells was determined by measuring the fluorescence of the supernatant. After assessing parallelism of dose-response curves, the percent cytotoxicity of ABP 501, adalimumab (US), or adalimumab (EU) test samples relative to the reference standard was determined using a 4-parameter logistic model fit (SoftMax Pro). Multiple lots $(\geq 10)$ of ABP 501, adalimumab (US), and adalimumab (EU) were tested, with three independent determinations for each lot. 


\subsection{Statistical Analysis}

For the TNF $\alpha$-binding (ELISA) assay and potency (U937 apoptosis inhibition) assay, similarity was assessed using statistical equivalence. Under this approach, similarity was achieved when the confidence interval $(\mathrm{CI})$ for the difference in means between the products was contained within an equivalence acceptance criterion of \pm 1.5 times the standard deviation of the adalimumab lots tested. For secondary mechanisms of action, including ADCC, CDC, Fc $\gamma$ RIIIa (158V) binding, and FcRn binding, results were considered statistically similar when $90 \%$ of the ABP 501 lots fell within a pre-defined quality range established based on the adalimumab lots tested; the quality range was defined as the mean of the adalimumab lots tested \pm 3 standard deviations. For characterization assays, three lots each of ABP 501 and adalimumab were tested, and with this number of tested lots, statistical assessment of similarity was not performed, and similarity was determined by a qualitative comparison of the results.

\section{Results}

\subsection{Binding to Soluble and Transmembrane TNF $\alpha$ is Similar Between ABP 501 and Adalimumab}

An ELISA assay was performed to compare the binding of ABP 501, adalimumab (US), and adalimumab (EU) to (immobilized) recombinant sTNF $\alpha$. Relative binding to TNF $\alpha$ was similar between the tested mAbs (Fig. 2) and the mean relative binding by ABP 501 was statistically similar to the binding observed for adalimumab, based on equivalence acceptance criteria. Specifically, the mean ABP 501 relative binding was $108.10 \%$ and mean adalimumab (US) relative binding was $111.83 \%$, with the difference between means of $-3.74 \%(90 \% \mathrm{CI}-11.03$ to 3.55). The equivalence acceptance criterion (EAC) for the difference was $\pm 15.02 \%$ for adalimumab (US) and thus the products are considered statistically equivalent. The mean relative binding for adalimumab (EU) was $111.33 \%$, with a difference from ABP 501 of $-3.23 \%(90 \% \mathrm{CI}$ -9.39 to 2.93 ). The EAC for adalimumab (EU) similarity was a $\pm 15.58 \%$ difference, and thus ABP 501 is also statistically equivalent to adalimumab (EU).

To further characterize the binding characteristics, biacore SPR was used to provide a comparison of the binding kinetics of ABP 501, adalimumab (US), and adalimumab (EU) to sTNF $\alpha$. The on rates, off rates and $K_{\mathrm{d}}$ for soluble TNF $\alpha$ binding to three different lots each of ABP 501, adalimumab (US), and adalimumab (EU) were similar (Table 1). The equilibrium binding affinity of ABP 501 to sTNF $\alpha$ was 52,48 , and $51 \mathrm{pM}$ for the three lots tested. The

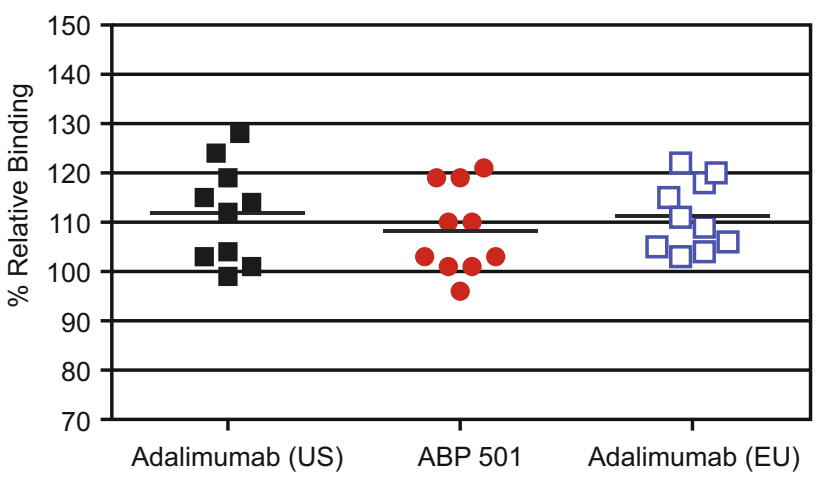

Fig. 2 Similarity of ABP 501, adalimumab (US), and adalimumab (EU) with respect to binding to sTNF $\alpha$. The relative binding values for ABP 501 (red), adalimumab (US) (black), and adalimumab (EU) (blue) to immobilized recombinant TNF $\alpha$ in an ELISA format are shown. Each point represents results from testing a unique lot. The lines represent the mean relative binding for that sample. Adalimumab (EU) EU-authorized adalimumab, adalimumab (US) FDA-licensed adalimumab, ELISA enzyme-linked immunosorbent assay, $s T N F \propto$ soluble tumor necrosis factor $\alpha$

Table 1 Binding kinetics of ABP 501, adalimumab (US), and adalimumab (EU) to sTNF $\alpha$. Kinetics of binding were determined by surface plasmon resonance, testing three unique lots of each test $\mathrm{mAb}$

\begin{tabular}{llll}
\hline Sample & On rate $k_{\mathrm{a}}(1 / \mathrm{ms})$ & Off rate $k_{\mathrm{d}}(1 / \mathrm{s})$ & $K_{\mathrm{d}}(\mathrm{pM})$ \\
\hline ABP 501 & $7.62 \mathrm{E}+5$ & $3.94 \mathrm{E}-5$ & 52 \\
ABP 501 & $7.69 \mathrm{E}+5$ & $3.73 \mathrm{E}-5$ & 48 \\
ABP 501 & $8.35 \mathrm{E}+5$ & $4.28 \mathrm{E}-5$ & 51 \\
Adalimumab (US) & $7.45 \mathrm{E}+5$ & $3.94 \mathrm{E}-5$ & 53 \\
Adalimumab (US) & $8.34 \mathrm{E}+5$ & $3.98 \mathrm{E}-5$ & 48 \\
Adalimumab (US) & $8.12 \mathrm{E}+5$ & $4.27 \mathrm{E}-5$ & 53 \\
Adalimumab (EU) & $8.08 \mathrm{E}+5$ & $4.38 \mathrm{E}-5$ & 54 \\
Adalimumab (EU) & $8.58 \mathrm{E}+5$ & $3.90 \mathrm{E}-5$ & 46 \\
Adalimumab (EU) & $8.65 \mathrm{E}+5$ & $4.44 \mathrm{E}-5$ & 51 \\
\hline
\end{tabular}

Adalimumab (EU) EU-authorized adalimumab, Adalimumab (US) FDA-licensed adalimumab, $k_{a}$ association rate constant, $k_{d}$ dissociation rate constant, $K_{d}$ dissociation equilibrium binding constant, $M$ molar, $m A b$ monoclonal antibody, $m s$ milliseconds, $s$ seconds, $s T N F \alpha$ soluble tumor necrosis factor $\alpha$

equilibrium binding affinity was 53,48 , and $53 \mathrm{pM}$ for the tested lots of adalimumab (US), and was 54,46 , and $51 \mathrm{pM}$ for the tested lots of adalimumab (EU) (Table 1).

Adalimumab can bind mbTNF $\alpha$ as well as sTNF $\alpha$, ultimately blocking signaling induced by mbTNF $\alpha$ or potentially mediating cellular effects directly by engaging mbTNF $\alpha$. Modulation of cellular activities subsequent to binding mbTNF $\alpha$ have been proposed to be relevant to efficacy in inflammatory bowel disease [18]. In order to further characterize the binding characteristics, similarity in binding to mbTNF $\alpha$ was determined in a competitive cell-based assay using MT-3 cells [17]. The mean (from three independent experiments) percent relative binding to 


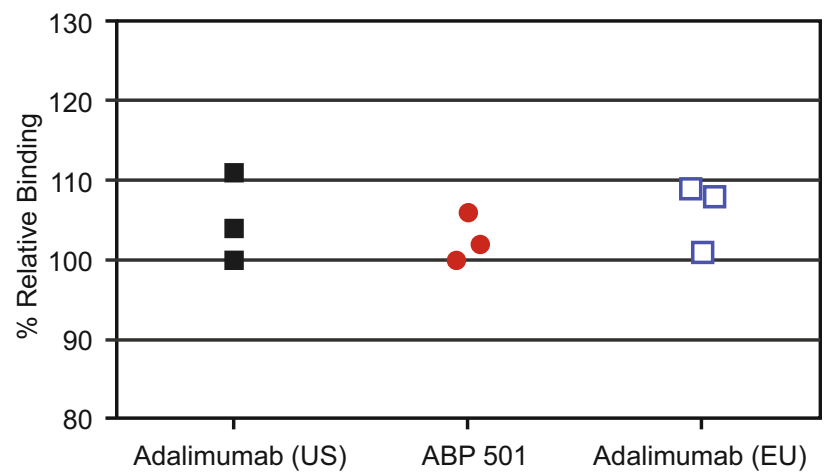

Fig. 3 Similarity of ABP 501, adalimumab (US), and adalimumab (EU) with respect to binding to $\mathrm{mbTNF} \alpha$. The relative binding values for ABP 501 (red), adalimumab (US) (black), and adalimumab (EU) (blue) in a competitive binding assay to mbTNF $\alpha$ using MT-3 cells are shown. Each point represents results from testing a unique lot. Adalimumab (EU) EU-authorized adalimumab, adalimumab (US) FDA-licensed adalimumab, $m b T N F \alpha$ transmembrane tumor necrosis factor $\alpha, M T-3$ Chinese hamster ovary cells expressing non-cleavable $\operatorname{mbTNF} \alpha$

mbTNF $\alpha$ for three tested lots of ABP 501 was 100-106 \%, for three lots of adalimumab (US) was 100-111\%, and for three lots of adalimumab (EU) was 101-109\% (Fig. 3). With the observed overlap in the results when comparing ABP 501 with adalimumab (US) and adalimumab (EU), the binding to mbTNF $\alpha$ was concluded to be similar.

\subsection{Neutralization of TNF $\alpha$ Bioactivity Is Similar Between ABP 501 and Adalimumab}

The primary mechanism of action of adalimumab is the inhibition of proinflammatory signaling induced by sTNF $\alpha$. $\mathrm{TNF} \alpha$ has been shown to induce apoptosis in cells, especially under conditions in which $\mathrm{NF \kappa B}$ activity is reduced [8]. As a well established measure of $\mathrm{TNF} \alpha$ activity in vitro, the potency of ABP 501 was compared with the potency of adalimumab (US) and adalimumab (EU) by testing the inhibition of TNF $\alpha$-induced apoptosis in U937 cells. Apoptosis was assessed as caspase 3/7 activation. As shown in Fig. 4a, the potency of ABP 501 is similar to that of adalimumab (US) and adalimumab (EU). The mean ABP 501 relative potency was $103.77 \%$ and mean adalimumab (US) relative potency was $105.50 \%$, with the difference between means of $-1.73 \%(90 \% \mathrm{CI}-5.17$ to 1.72), which is within the EAC of $\pm 8.64 \%$ for the difference from adalimumab (US). The mean relative potency for adalimumab (EU) was $102.83 \%$, with a difference from ABP 501 of $0.94 \%$ (90\% CI -4.42 to 6.29), which is within the $\pm 14.04 \%$ EAC for the difference from adalimumab (EU). Therefore, APB 501 is statistically equivalent to adalimumab (US) and to adalimumab (EU).

$\mathrm{TNF} \alpha$ is known to induce a proinflammatory cascade of cytokine and chemokine induction, which largely explains the effectiveness of TNF $\alpha$ inhibition in treating autoimmune disease. To further characterize the similarity in neutralization of $\mathrm{TNF} \alpha$, the ability of $\mathrm{ABP} 501$, adalimumab (US), and adalimumab (EU) to inhibit TNF $\alpha$-induced IL-8 secretion in HUVEC was assessed, testing three lots of each test mAb. ABP 501 inhibited TNF $\alpha$-induced IL-8 secretion from HUVEC with $\mathrm{EC}_{50}$ values ranging from 192 to $294 \mathrm{pM}$, which were of a similar range to that observed for adalimumab (US) (131-253 pM) and adalimumab (EU) (168-225 pM). Dose response results from a representative assay are shown in Fig. 4b. In order to confirm the specificity of ABP 501, adalimumab (US), and adalimumab (EU), the mAb samples were shown to be unable to inhibit LT $\alpha$-induced IL-8 production (Fig. $4 b$ ). The inhibition of chemokine induction, including specificity against LT $\alpha$, is similar between ABP 501, adalimumab (US), and adalimumab (EU).

In addition to inducing proinflammatory cytokine/chemokine production and apoptosis, TNF $\alpha$ can induce nonapoptotic cell death. As further characterization, the ability of ABP 501, adalimumab (US), and adalimumab (EU) to inhibit TNF $\alpha$-induced cell death in L929 cells was tested. Three lots of each $\mathrm{mAb}$ were compared. ABP 501 inhibited recombinant human $\mathrm{TNF} \alpha$-induced cell death with $\mathrm{EC}_{50}$ values ranging from 240 to $511 \mathrm{pM}$ for the three lots, compared with 284-544 pM for the lots of adalimumab (US) and 294-407 pM for the lots of adalimumab (EU). A representative dose-response curve from one lot of each of the test mAbs for the inhibition of TNF $\alpha$-induced cytotoxicity is presented in Fig. 4c. The ability of ABP 501, adalimumab (US), and adalimumab (EU) to inhibit TNF $\alpha$ induced cell death is similar.

\subsection{Fc Receptor Binding is Similar Between ABP 501 and Adalimumab}

Fc $\gamma$ Rs play a critical role in regulating immune responses. Signaling through this receptor family can result in cytokine release, modulation of cell activation, apoptosis, phagocytosis, and ADCC. Fc $\gamma$ RIa is the only high-affinity $\mathrm{Fc} \gamma \mathrm{R}$ able to bind monomeric IgG [19]. Crosslinking of Fc $\gamma$ RIa results in classical spleen tyrosine kinase (Syk)mediated downstream signaling [20], but the ultimate functional consequence of Fc $\gamma$ RIa signaling is not well understood, so the implications of this binding activity for clinical mechanism of action are not established. The similarity in binding to Fc $\gamma$ RIa was compared between ABP 501, adalimumab (US), and adalimumab (EU) in a competitive AlphaLISA binding assay. Mean (from three independent experiments) percent relative binding values for the Fc $\gamma$ RIa AlphaLISA binding assay ranged from 96 to $99 \%$ for the ABP 501 lots, 92-96\% for the adalimumab (US) lots, and 92-94\% for the adalimumab (EU) lots. 


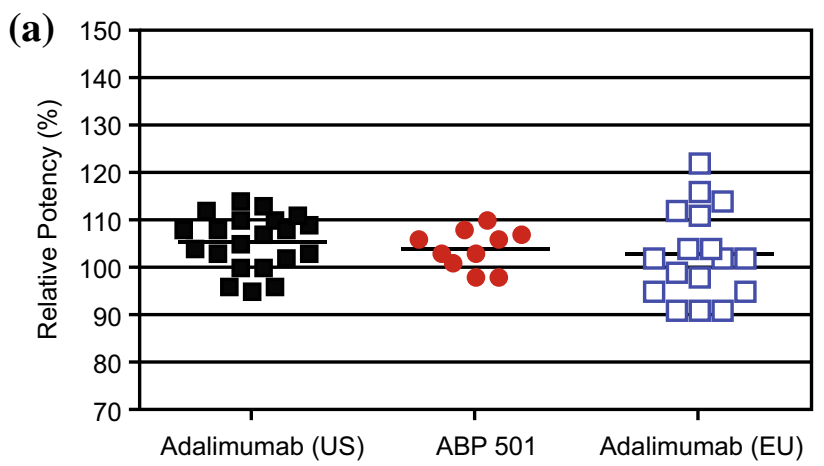

(b)
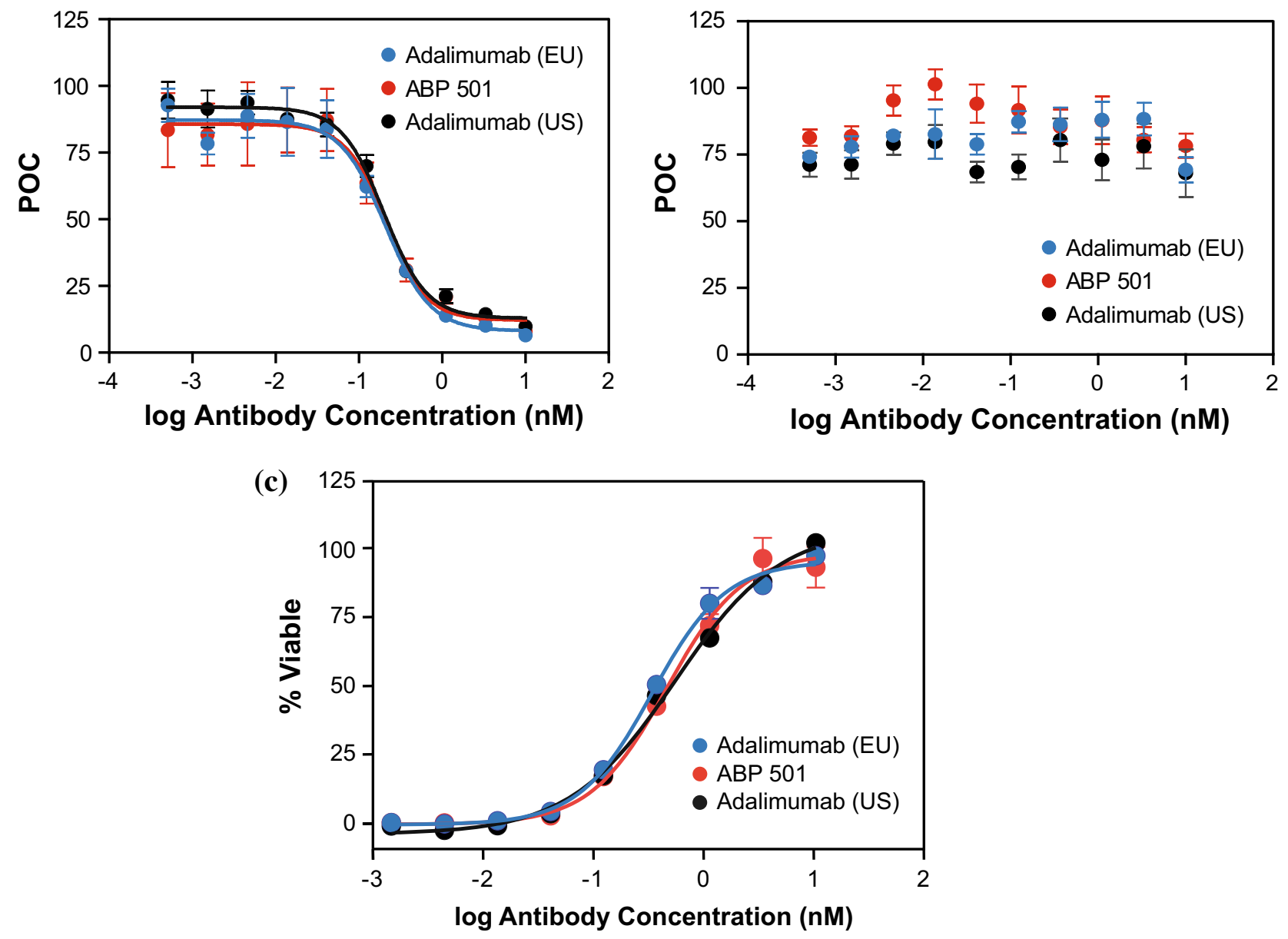

Fig. 4 Similarity of ABP 501, adalimumab (US), and adalimumab (EU) in $\mathrm{TNF} \alpha$-induced functional assays. a Inhibition of $\mathrm{TNF} \alpha-$ induced caspase activation in U937 by ABP 501 (red), adalimumab (US) (black), and adalimumab (EU) (blue). Each point represents results from testing a unique lot. The lines represent the mean relative potency for that sample. b Inhibition of human TNF $\alpha$ induced IL-8 secretion in HUVEC. Titration of ABP 501 (red), adalimumab (US) (black), and adalimumab (EU) (blue) against $3 \mathrm{ng} / \mathrm{mL}$ TNF $\alpha$-stimulated IL-8 production in HUVEC (left) or $10 \mathrm{ng} / \mathrm{mL} \mathrm{LT} \alpha$-stimulated IL-8 production in HUVEC (right). Results depicted are from a representative assay. Each point represents the mean POC of triplicates \pm SEM. c Inhibition of human TNF $\alpha$-induced cytotoxicity in L929 cells. Dose-responsive inhibition of $0.75 \mathrm{ng} / \mathrm{mL} \mathrm{TNF} \alpha$-stimulated cytotoxicity in L929 cells, showing a titration of ABP 501 (red), adalimumab (US) (black), and adalimumab (EU) (blue). Representative dose-response curves are shown with each point representing the mean percent viable cells for triplicates \pm SEM. Adalimumab $(E U)$ EU-authorized adalimumab, adalimumab (US) FDA-licensed adalimumab, HUVEC human umbilical vein endothelial cells, $I L-8$ interleukin-8, $L T \alpha$ lymphotoxin- $\alpha, P O C$ percent of control, SEM standard error of the mean, $T N F \alpha$ tumor necrosis factor $\alpha$ 
Representative (from one of three experiments) dose response overlay curves are shown in Fig. 5a. The results demonstrate that the relative Fc $\gamma$ RIa binding activities of ABP 501, adalimumab (US), and adalimumab (EU) are similar.

Fc $\gamma$ RIIa is a low-affinity $\mathrm{Fc} \gamma \mathrm{R}$ expressed on myeloid and some lymphoid cells, including mast cells, macrophages, monocytes, dendritic cells, neutrophils, and platelets. There are two allelic variants of Fc $\gamma$ RIIa, expressing either arginine (high responder, low affinity) or histidine (low responder, high affinity) at position 131 [19]. Since Fc $\gamma$ RIIa can mediate phagocytosis and platelet activation, and the affinity of Fc $\gamma$ RIIa binding can be impacted by post-translational modifications and aggregate levels, an Fc $\gamma$ RIIa $(131 \mathrm{H})$ competitive AlphaLISA binding assay was used to compare ABP 501, adalimumab (US), and adalimumab (EU). The mean (from three independent experiments) percent relative binding values for the Fc $\gamma$ RIIa AlphaLISA binding assay ranged from 95 to $107 \%$ for the ABP 501 lots, 101-105\% for the adalimumab (US) lots, and $96-100 \%$ for the adalimumab (EU) lots. Representative (one of three experiments) dose-response curves from the Fc $\gamma$ RIIa binding assay are shown in Fig. 5b. The results demonstrate that the relative Fc $\gamma$ RIIa binding activities of ABP 501, adalimumab (US), and adalimumab (EU) are similar.

Fc $\gamma$ RIIIa is a pro-inflammatory receptor expressed on human natural killer cells, and is involved in the induction of ADCC. A genetic polymorphism in Fc $\gamma$ RIIIa results in expression of valine $(\mathrm{V}$, high affinity) or phenylalanine $(\mathrm{F}$, low affinity) at amino acid 158. Binding of ABP 501, adalimumab (US), and adalimumab (EU) to both variants was tested. The binding of ABP 501, adalimumab (US), and adalimumab (EU) to Fc $\gamma$ RIIIa $(158 \mathrm{~V})$, the high-affinity allotype, was determined for $\geq 10$ lots of each test mAb. Similar activity was observed as illustrated in the representative dose response curve in Fig. 5c. The mean (from three independent experiments) percent relative binding values for the Fc $\gamma$ RIIIa (158V) AlphaLISA binding assay ranged from 67 to $113 \%$ for the ABP 501 lots, 76-114\% for the adalimumab (US) lots, and $86-104 \%$ for the adalimumab (EU) lots. Furthermore, ABP 501 was statistically similar in that the relative binding values of the ABP 501 lots fell within the quality range established based on the adalimumab lots tested.

Human monoclonal antibodies to $\mathrm{TNF} \alpha$, including adalimumab, have been shown to form higher-order complexes with TNF $\alpha[21,22]$. These complexes can form because bivalent antibodies can bind two different $\mathrm{TNF} \alpha$ molecules and trimeric TNF $\alpha$ molecules can bind multiple antibody molecules. The higher-order complexes that form can increase the apparent affinity to $\mathrm{Fc}$ receptors involved in mediating effector functions due to the increase in avidity. In order to assess the impact of antibody/TNF $\alpha$ complex formation on the Fc effector function potential of ABP 501, adalimumab (US), and adalimumab (EU), the Fc $\gamma$ RIIIa AlphaLISA binding assay was performed in the presence of an equimolar concentration of recombinant $\mathrm{STNF} \alpha$, testing a single lot of each $\mathrm{mAb}$. In the presence of $\mathrm{TNF} \alpha$, the mean (three independent experiments) percent relative binding to Fc $\gamma$ RIIIa (158V) was $108 \%$ for ABP 501, $101 \%$ for adalimumab (US), and $113 \%$ for adalimumab (EU). Representative dose-response curves from the binding assays with and without TNF $\alpha$ addition are shown in Fig. 5c. As expected, an affinity shift is observed in the presence of TNF $\alpha$, but the binding of ABP 501, adalimumab (US), and adalimumab (EU) are similar to each other in each experiment.

Relative binding to the Fc $\gamma$ RIIIa (158F) allotype was also determined. Mean percent relative binding in the Fc $\gamma$ RIIIa (158F) AlphaLISA assay (in the absence of $\mathrm{TNF} \alpha$ ) ranged from 73 to $93 \%$ for the three ABP 501 lots; $83-95 \%$ for the three adalimumab (US) lots; and 88-98\% for the three adalimumab (EU) lots. Representative (one experiment of the three performed) dose-response curves from the binding assay are shown in Fig. 5d. The results demonstrate that the relative binding of ABP 501, adalimumab (US), and adalimumab (EU) to Fc $\gamma$ RIIIa (158F) is similar.

The FcRn binds IgG heavy chains in the Fc region under mildly acidic conditions (pH 6) and releases IgG at neutral $\mathrm{pH}$ (7.4). It is through this highly $\mathrm{pH}-$-dependent interaction that FcRn mediates IgG homeostasis in human adults through recycling of IgG back into the serum. A cell-based FcRn binding assay using a variant of the human embryonic kidney cell line overexpressing human FcRn (293T7A1) was used to test the binding of the Fc moiety of ABP 501, adalimumab (US), and adalimumab (EU) to FcRn. Mean (from three independent experiments) percent relative binding of ABP 501, adalimumab (US), and adalimumab (EU) to $\mathrm{FcRn}$ was similar, with relative binding values ranging from 86 to $101 \%$ for the ABP 501 lots, $91-114 \%$ for the adalimumab (US) lots, and $81-116 \%$ for the adalimumab (EU) lots. Representative (one of three experiments performed) dose-response overlay results are shown in Fig. 5e. Statistical evaluation of the results demonstrate that the relative binding to $\mathrm{FcRn}$ is similar between ABP 501, adalimumab (US), and adalimumab (EU), since relative binding of the ABP 501 lots fell within the quality range established based on the adalimumab lots tested. 

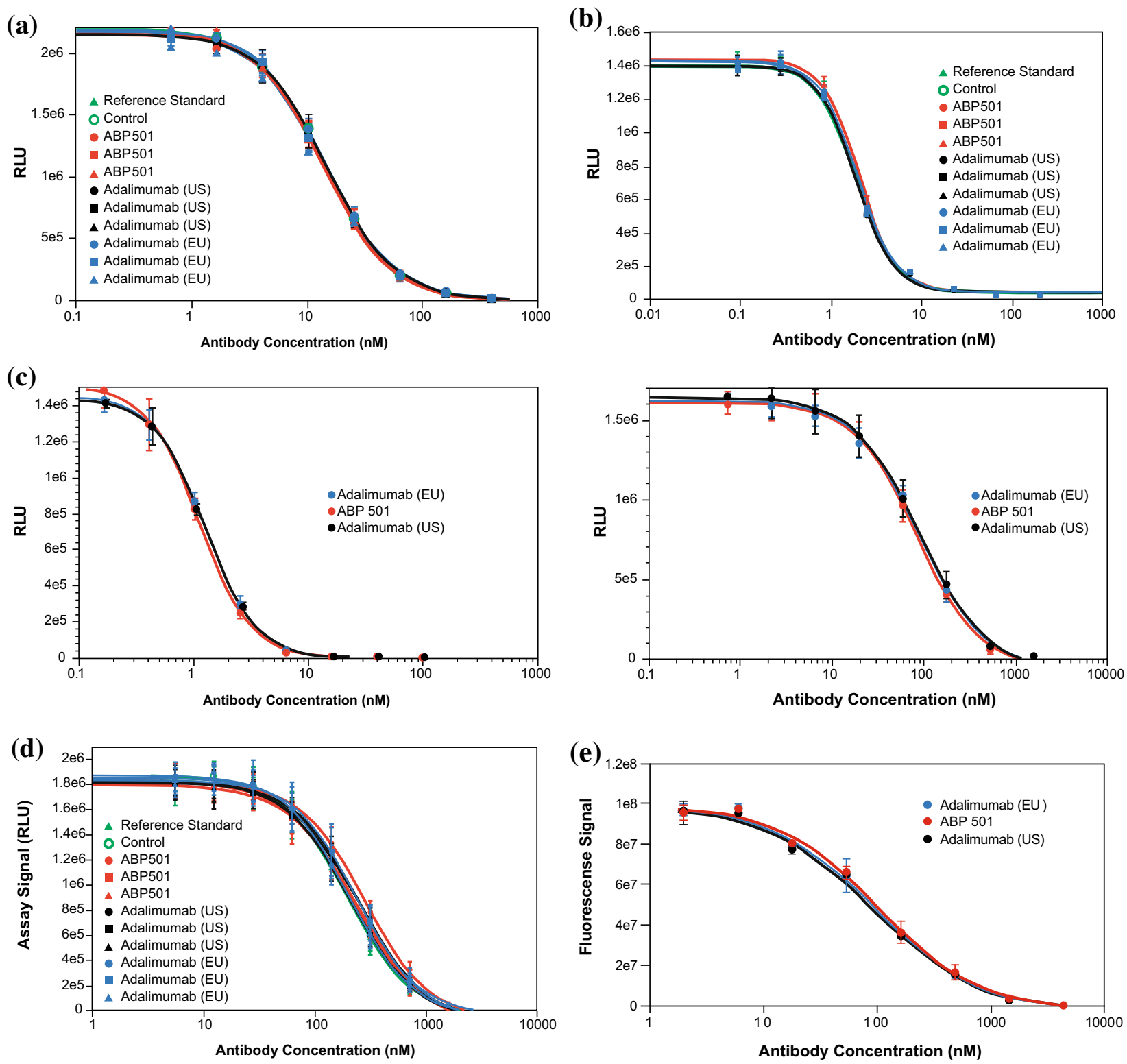

Fig. 5 Similarity of ABP 501, adalimumab (US) and adalimumab (EU) in Fc-gamma receptor $(\mathrm{Fc} \gamma \mathrm{R})$ binding. a Representative nonconstrained dose-response curves of reference standard and control (green), ABP 501 (red), adalimumab (US) (black), and adalimumab (EU) (blue) showing binding to Fc $\gamma$ RIa. Three different lots of each $\mathrm{mAb}$ were tested and each dose point represents the mean of three intra-assay replicates \pm standard deviation. A total of three independent assays were conducted and the mean percent relative activity is reported (see text). A dose response from a single assay of the three is presented here as representative data. b Representative nonconstrained dose-response curves of reference standard and control (green), ABP 501 (red), adalimumab (US) (black), and adalimumab (EU) (blue) showing binding to Fc $\gamma$ RIIa. Three different lots of each $\mathrm{mAb}$ were tested and each dose point represents the mean of three intra-assay replicates \pm standard deviation. A total of three independent assays were conducted, and the mean percent relative activity is reported (see text). A dose response from a single assay of the three is presented here as representative data. c Representative nonconstrained dose-response curves of ABP 501 (red), adalimumab (US)

(black), and adalimumab (EU) (blue) showing Fc $\gamma$ RIIIa (158V) binding with (left) and without (right) $\mathrm{TNF} \alpha$. Each dose point represents the mean of three intra-assay replicates \pm standard deviation. d Representative nonconstrained dose-response curves of reference standard and control (green), ABP 501 (red), adalimumab (US) (black), and adalimumab (EU) (blue) showing binding to Fc $\gamma$ RIIIa (158F). Three different lots of each mAb were tested and each dose point represents the mean of three intra-assay replicates \pm standard deviation. A total of three independent assays were conducted, and the mean percent relative activity is reported (see text). A dose response from a single assay of the three is presented here as representative data. e Representative nonconstrained doseresponse curves of ABP 501 (red), adalimumab (US) (black), and adalimumab (EU) (blue) showing binding to FcRn. Each point is a mean of three replicates \pm standard deviation. Adalimumab $(E U) \mathrm{EU}$ authorized adalimumab, adalimumab (US) FDA-licensed adalimumab, FcRn Fc neonatal receptor, $m A b$ monoclonal antibody, $R L U$ relative luminescence units, TNF $\alpha$ tumor necrosis factor $\alpha$ 


\subsection{Induction of Effector Function is Similar Between ABP 501 and Adalimumab}

Adalimumab is able to mediate ADCC in vitro $[9,17,23]$. Although the contribution of ADCC activity to clinical efficacy is unclear, it is important to characterize all activities of the candidate $\mathrm{mAb}$, especially those that can be affected by differences in post-translational modifications, such as glycosylation. The ability of ABP 501 to induce ADCC was assessed using MT- 3 cells as target cells, and NK-92M1 cells stably transfected with human Fc $\gamma$ RIIIa $(158 \mathrm{~V})$ as effector cells. Mean (three independent experiments) percent relative ADCC activities were determined for ABP 501, adalimumab (US), and adalimumab (EU). As shown in Fig. 6a with the quality range depicted by dotted lines, statistical similarity was demonstrated since all of the ABP 501 lots fell within the quality range established based on the adalimumab (US) lots tested.

Another mechanism for inducing cell death is the induction of CDC in cells expressing mbTNFa. A comparison of the CDC activity of ABP 501 to that of adalimumab (US) and adalimumab (EU) using MT-3 cells as target cells was conducted. Mean (three independent experiments) percent relative CDC activities were similar (Fig. 6b) and since the ABP 501 relative activity results were within the quality range established by the adalimumab (US) lots, the activity is considered statistically similar.

(a)

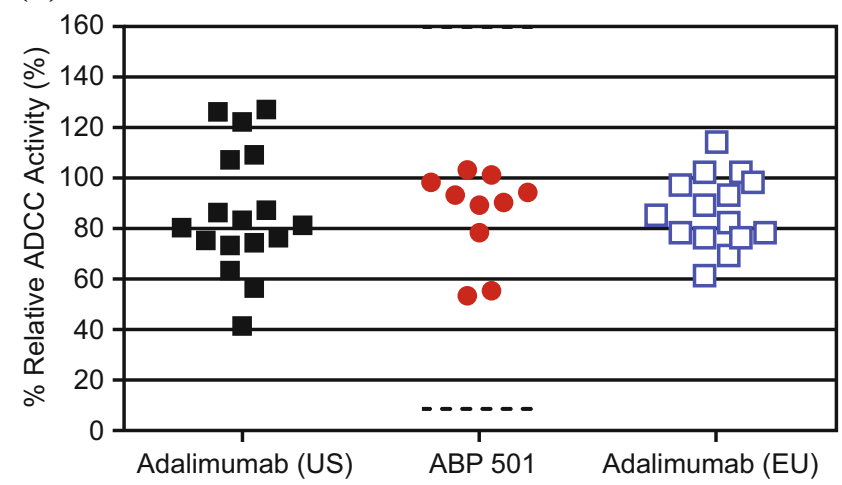

Fig. 6 Similarity of ABP 501, adalimumab (US), and adalimumab (EU) in effector function activity. a Induction of ADCC by ABP 501 (red), adalimumab (US) (black), and adalimumab (EU) (blue). Each point represents results from testing a unique lot. The dotted lines represent the quality range established based on the adalimumab (US) lots tested (mean \pm 3 standard deviations). Adalimumab (EU) EUauthorized adalimumab, adalimumab (US) FDA-licensed adalimumab, $A D C C$ antibody-dependent cell-mediated cytotoxicity.

\section{Discussion}

$\mathrm{TNF} \alpha$ is a pleiotropic cytokine that is able to mediate diverse cellular functions in order to finely control the immune response in vivo. Among its cellular functions, $\mathrm{TNF} \alpha$ is able to induce cytokines, chemokines, proliferation, and also cell death. The induction of pro-inflammatory versus death signals depends upon the molecular context of the responding cell, and specifically whether $\mathrm{NF \kappa B}$ is involved [8]. Adding to the complexity of signaling, it is also reported that TNF $\alpha$ exists in both soluble and transmembrane forms, with differing reported activities in each case. Not surprisingly, given the multifaceted nature of TNF $\alpha$, many diverse mechanisms of action have been reported for adalimumab and other approved antiTNF agents [7, 10]. Therefore, a comprehensive assessment of the bioactivity of adalimumab should include assessment of multiple in vitro endpoints (NFKB-dependent and NFkB-independent) and should include binding to both soluble and transmembrane TNF $\alpha$. ABP 501 has been shown to be similar to adalimumab in its ability to neutralize TNF $\alpha$-induced caspase activation, chemokine production, and cytotoxicity, functions inclusive of both

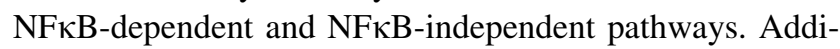
tionally, similarity has been demonstrated in binding to soluble and transmembrane $\mathrm{TNF} \alpha$, including an assessment of binding kinetics by SPR. Demonstrating no gain of function is also an important aspect in biosimilar

(b)

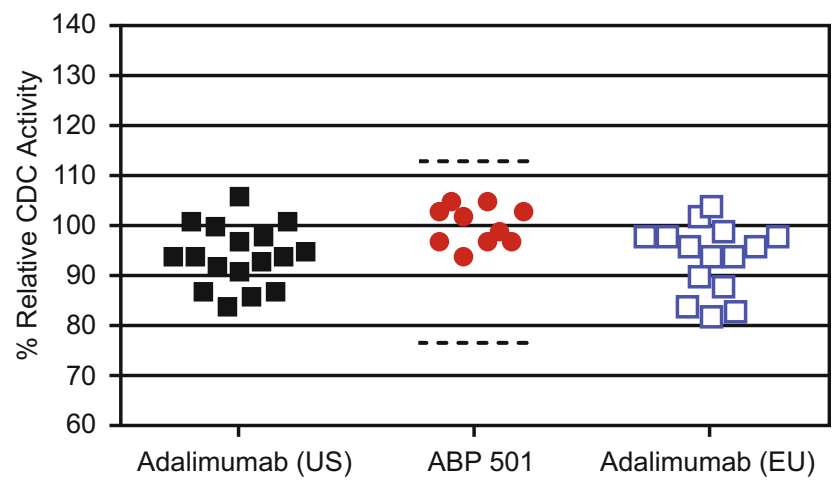

b Induction of CDC by ABP 501 (red), adalimumab (US) (black), and adalimumab (EU) (blue). Each point represents results from testing a unique lot. The dotted lines represent the quality range established based on the adalimumab (US) lots tested (mean \pm 3 standard deviations). Adalimumab (EU) EU-authorized adalimumab, adalimumab (US) FDA-licensed adalimumab, CDC complementdependent cytotoxicity 
development and the results presented here show that both ABP 501 and adalimumab are unable to neutralize the bioactivity of LT $\alpha$, the most closely related cytokine to TNF $\alpha$. The methods used to demonstrate functional similarity in the fragment antigen-binding (Fab) region of the $\mathrm{mAb}$ were shown to be able to discriminate a thermally degraded sample (data not shown), demonstrating that the utilized assays are sensitive to detect differences in activity, if they did exist.

It is well established that $\operatorname{IgG} 1 \mathrm{mAbs}$ are efficient mediators of effector function, and are able to bind to many of the known Fc $\gamma$ Rs. A sensitive comparison of these Fcdependent activities is important in biosimilar development, since glycosylation and other product quality attribute differences are to be expected between biosimilars and reference products due to inherent cell line differences (even in the same cell expression system) and the proprietary nature of manufacturing. ABP 501 has been shown to be similar to adalimumab with respect to binding to a panel of Fc receptors, including Fc $\gamma$ RIa, Fc $\gamma$ RIIa, Fc $\gamma$ RIIIa (158V) (with and without TNF $\alpha$ ), and Fc $\gamma$ RIIIa (158F). Importantly, effector function activation (ADCC and CDC) was also demonstrated to be similar between ABP 501 and adalimumab using highly sensitive methods. The ADCC and CDC methods have been demonstrated to be sensitive to detect differences in the glycan profile of the mAbs (data not shown). Although effector function is not known to be directly associated with the clinical efficacy of adalimumab, it cannot be ruled out conclusively, especially in inflammatory bowel disease. The efficacy of the TNF $\alpha$ neutralizing Fab certolizumab is reduced in Crohn's disease relative to the efficacy observed with adalimumab $[24,25]$, which suggests that Fc-mediated effector functions may be important. Binding of mAbs to FcRn affects clearance, so a similarity assessment of biosimilars should also include sensitive methods to assess binding to FcRn. ABP 501 was shown to have similar binding to FcRn as compared with adalimumab.

Given the similarity in analytical characteristics as reported separately (Liu et al. [16]) and biofunctional activity demonstrated here, ABP 501 is expected to be clinically similar to the adalimumab reference product without any clinically meaningful differences. Clinical data will help to confirm the safety and efficacy profile of ABP 501. In a phase I human pharmacokinetic study, ABP 501 has been shown to be similar to adalimumab (US) and adalimumab (EU) [26, 27]. Clinical studies designed to assess the similarity of ABP 501 relative to the adalimumab reference product for the treatment of moderate to severe plaque psoriasis $[28,29]$ and moderate to severe rheumatoid arthritis $[30,31]$ have been completed.

\section{Conclusion}

During the stepwise development of a biosimilar mAb, it is crucial that analytical and biofunctional similarity to the reference product be demonstrated with sensitive, state-ofthe-art methods that exhaustively examine all potential regions of the $\mathrm{mAb}$ and mechanisms of action. This is often an iterative process in which analytical similarity is tested and then further explored in preclinical biofunctional tests to determine whether minor differences, should they exist, result in differences in the biological activity of the molecule (Fig. 1). Confidence in the similarity of the molecule at this foundational step allows for a reduction in residual uncertainty as the molecule progresses into clinical testing for equivalence $[2,13,15,32]$. The current data, as presented, demonstrate that ABP 501 is similar to adalimumab (US) and adalimumab (EU) with respect to multiple analytical (Liu et al. [16], includes additional data on potency as measured in multiple lots) and biofunctional parameters. The biofunctional parameters tested include an assessment of both binding and function within both the Fab and Fc portions of the mAb.

Acknowledgments The authors thank Abhishek Mathur for scientific and technical contributions to the reported data and Jennifer Liu, Jill Crouse-Zeineddini, and Scott Kuhns for guidance on overall similarity assessment strategy. Medical writing and editorial support was provided by Rozena Varghese, RPh, PharmD, of MedVal Scientific Information Services, LLC, Skillman, NJ, USA, under the guidance of Monica Ramchandani, Ph.D., Amgen Inc.

Author contributions Jyoti Velayudhan is the lead author and subject expert for effector function testing. Teresa L. Born is the corresponding author and is responsible for the overall design and execution of the studies. Yuh-feng Chen is the subject expert for potency assessment and he contributed to the authoring and reviewing of this manuscript. Amanda Rohrbach, Christina Pastula, and Gwen Maher are the subject experts for binding assays and they contributed to the authoring and reviewing of this manuscript. Heather Thomas is the subject expert for sTNF characterization assays and she contributed to the authoring and reviewing of this manuscript. Ryan Brown is the subject expert for surface plasmon resonance analysis and he contributed to the authoring and reviewing of this manuscript.

\section{Compliance with Ethical Standards}

Amgen Inc. conducted and funded all analyses. Amgen Inc. also provided funding for medical writing and editorial support to MedVal Scientific Information Services, LLC, Skillman, NJ, USA. This article does not contain any studies with human participants or animals performed by any of the authors. This paper was prepared according to the ICMJE Uniform Requirements and the International Society for Medical Publication Professionals' "Good Publication Practice for Communicating Company-Sponsored Medical Research: The GPP2 Guidelines". 
Conflict of interest Jyoti Velayudhan, Amanda Rohrbach, Christina Pastula, Heather Thomas, and Ryan Brown are former employees and stockholders of Amgen, Inc. Yuh-feng Chen, Gwen Maher, and Teresa L. Born are employees and stockholders of Amgen, Inc.

Open Access This article is distributed under the terms of the Creative Commons Attribution-NonCommercial 4.0 International License (http://creativecommons.org/licenses/by-nc/4.0/), which permits any noncommercial use, distribution, and reproduction in any medium, provided you give appropriate credit to the original author(s) and the source, provide a link to the Creative Commons license, and indicate if changes were made.

\section{References}

1. US Centers for Medicare and Medicaid Services. National health expenditures 2013 highlights. http://www.cms.gov/ResearchStatistics-Data-and-Systems/Statistics-Trends-and-Reports/ NationalHealthExpendData/Downloads/highlights.pdf. Accessed 10 Apr 2015.

2. Zelenetz AD, Ahmed I, Braud EL, Cross JD, Davenport-Ennis N, Dickinson BD, et al. NCCN Biosimilars White Paper: regulatory, scientific, and patient safety perspectives. J Natl Compr Cancer Netw. 2011;9(Suppl. 4):S1-22.

3. US Department of Health and Human Services, Food and Drug Administration, Center for Drug Evaluation and Research, Center for Biologics Evaluation and Research. Scientific considerations in demonstrating biosimilarity to a reference product. Guidance for industry. http://www.fda.gov/downloads/Drugs/Guidance ComplianceRegulatoryInformation/Guidances/UCM291128.pdf. Accessed 12 May 2015.

4. US Department of Health and Human Services, Food and Drug Administration, Center for Drug Evaluation and Research, Center for Biologics Evaluation and Research. Guidance for industry: clinical pharmacology data to support a demonstration of biosimilarity to a reference product. http://www.fda.gov/ downloads/Drugs/GuidanceComplianceRegulatoryInformation/ Guidances/UCM397017.pdf. Accessed 25 Feb 2015.

5. European Medicines Agency. Guideline on similar biological medicinal products. http://www.ema.europa.eu/docs/en_GB/ document_library/Scientific_guideline/2014/10/WC500176768. pdf. Accessed 3 Apr 2015.

6. Humira (adalimumab) injection [prescribing information]. North Chicago: AbbVie Inc.; September 1, 2015.

7. Tracey D, Klareskog L, Sasso EH, Salfeld JG, Tak PP. Tumor necrosis factor antagonist mechanisms of action: a comprehensive review. Pharmacol Ther. 2008;117(2):244-79.

8. Pimentel-Muinos FX, Seed B. Regulated commitment of TNF receptor signaling: a molecular switch for death or activation. Immunity. 1999;11(6):783-93.

9. Mitoma H, Horiuchi T, Tsukamoto H, Tamimoto Y, Kimoto Y, Uchino A, et al. Mechanisms for cytotoxic effects of anti-tumor necrosis factor agents on transmembrane tumor necrosis factor alpha-expressing cells: comparison among infliximab, etanercept, and adalimumab. Arthritis Rheum. 2008;58(5):1248-57.

10. Peake STC, Bernardo D, Mann ER, Al-Hassi HO, Knight SC, Hart AL. Mechanisms of action of anti-tumor necrosis factor alpha agents in Crohn's disease. Inflamm Bowel Dis. 2013;19(7):1546-55.

11. Jefferis R. Glycosylation as a strategy to improve antibody-based therapeutics. Nat Rev Drug Discov. 2009;8(3):226-34.

12. Liu J, Hutterer K, Eris T, Abel J, Jiang Y, Li C, et al. Analytical similarity assessment of a biosimilar to adalimumab. American
Association of Pharmaceutical Scientists, November 2-6, 2014, San Diego, CA.

13. Feagan BG, Choquette D, Ghosh S, Gladman DD, Ho V, Meibohm B, et al. The challenge of indication extrapolation for infliximab biosimilars. Biologicals. 2014;42(4):177-83.

14. Weise M, Kurki P, Wolff-Holz E, Bielsky MC, Schneider CK. Biosimilars: the science of extrapolation. Blood. 2014;124(22): 3191-6.

15. Ebbers HC. Biosimilars: in support of extrapolation of indications. J Crohns Colitis. 2014;8(5):431-5.

16. Liu J, et al. Assessing analytical similarity of proposed Amgen biosimilar ABP 501 to adalimumab. BioDrugs. doi: 10.1007/ s40259-016-0184-3.

17. Arora T, Padaki R, Liu L, Hamburger AE, Ellison AR, Stevens $\mathrm{SR}$, et al. Differences in binding and effector functions between classes of TNF antagonists. Cytokine. 2009;45(2):124-31.

18. Horiuchi T, Mitoma H, Harashima S, Tsukamoto H, Shimoda T. Transmembrane TNF-a: structure, function and interaction with anti-TNF agents. Rheumatology. 2010;49(7):1215-28.

19. Rosales C, Uribe-Querol E. Fc receptors: cell activators of antibody functions. Adv Biosci Biotechnol. 2013;4:21-33.

20. van der Poel CE, Spaapen RM, van de Winkel JGJ, Leusen JHW. Functional characteristics of the high affinity $\mathrm{IgG}$ receptor, FcgRI. J Immunol. 2011;186(5):2699-704.

21. Kohno T, Tam LT, Stevens SR, Louie JS. Binding characteristics of tumor necrosis factor receptor-Fc fusion proteins vs anti-tumor necrosis factor mAbs. J Invest Dermatol Symp Proc. 2007;12(1): $5-8$.

22. Santora LC, Kaymakcalan Z, Sakorafas P, Krull IS, Grant K. Characterization of noncovalent complexes of recombinant human monoclonal antibody and antigen using cation exchange, size exclusion chromatography, and BIAcore. Analyt Biochem. 2001;299(2):119-29.

23. Nesbitt A, Fossati G, Bergin M, Stephens P, Stephens S, Foulkes $\mathrm{R}$, et al. Mechanism of action of certolizumab pegol (CDP870): in vitro comparison with other anti-tumor necrosis factor alpha agents. Inflamm Bowel Dis. 2007;13(11):1323-32.

24. Stidham RW, Lee TC, Higgins PD, Deshpande AR, Sussman DA, Singal AG, et al. Systematic review with network meta-analysis: the efficacy of anti-TNF agents for the treatment of Crohn's disease. Aliment Pharmacol Ther. 2014;39(12):1349-62.

25. Hazlewood GS, Rezaie A, Borman M, Panaccione R, Ghosh S, Seow $\mathrm{CH}$, et al. Comparative effectiveness of immunosuppressants and biologics for inducing and maintaining remission in Crohn's disease: a network meta-analysis. Gastroenterology. 2015;148(2):344-54.

26. Kaur PP, Chow V, Zhang N, Moxness M, Markus R. Pharmacokinetic equivalence of ABP 501 relative to adalimumab: results from a randomized, single-blind, single-dose, parallel group study in healthy subjects [abstract]. Arthritis Rheumatol. 2014;66(10 Suppl.):S661-2.

27. Kaur P, Chow V, Zhang N, Moxness M, Markus R. A randomized, single-blind, single-dose, parallel group study in healthy subjects to demonstrate pharmacokinetic equivalence of biosimilar candidate ABP 501 and adalimumab (EU) [abstract]. Ann Rheum Dis. 2014;73(Suppl. 2):479.

28. Papp K, Strober B, Bachelez H, Costanzo A, Weglowska J, Zhang N, et al. Results from a multicenter, phase 3 study evaluating efficacy and safety of ABP 501 compared with adalimumab in subjects with moderate to severe plaque psoriasis. European Academy of Dermatology and Venerology, October 7-11, 2015, Copenhagen, Denmark.

29. Strober B, Armstrong A, Gordon K, Gottlieb A, Greenberg JD, Koo J, et al. Evaluation of efficacy and safety of ABP 501 in a phase 3 study in subjects with moderate to severe plaque 
psoriasis: 52-week results. American Academy of Dermatology, March 4-8, 2016, Washington DC.

30. Cohen SB, Genovese MC, Choy EH, Perez-Ruiz F, Pablos JL, Zhang N, et al. Randomized, double-blind, phase 3 study of efficacy and safety of ABP 501 compared with adalimumab in subjects with moderate to severe rheumatoid arthritis [abstract]. Arthritis Rheumatol. 2015;67(Suppl. 10):2054.

31. Matsumoto AK, Pavelka K, Rizzo W, Gupta R, Shergy W, Haycaj P, et al. Secondary efficacy endpoints: sesults from a phase 3 study comparing ABP 501 with adalimumab in subjects with moderate to severe rheumatoid arthritis [abstract]. Arthritis Rheumatol. 2015;67(Suppl. 10):2757.

32. Bandyopadhyay S, Mahajan M, Mehta T, Singh AK, Parikh A, Gupta AK. Physicochemical and functional characterization of a biosimilar adalimumab ZRC-3197. Biosimilars. 2015;5:1-18. 